\title{
SOME PROPERTIES OF RATIONAL TRANSFER FUNCTIONS AND THEIR LAPLACE TRANSFORMATIONS*
}

\author{
BY \\ ARMEN H. ZEMANIAN \\ College of Engineering, New York University
}

Introduction. The behavior of a fixed, linear system at a pair of output terminals due to a signal impressed at a pair of input terminals is determined by the transfer function between these two terminals. More precisely, this transfer function $Z(s)$ is the ratio of the Laplace transform of the output function of time to the Laplace transform of the input function of time. Moreover, if the system is lumped and finite, the transfer function will be a rational function of the complex frequency variable $s=\sigma+j \omega$ and may be written as the ratio of two polynomials in $s$

$$
Z(s)=K \frac{s^{n}+a_{n-1} s^{n-1}+\cdots+a_{0}}{s^{m}+b_{m-1} s^{m-1}+\cdots+b_{0}}=K \frac{N(s)}{D(s)} .
$$

The constant multiplier $K$ and the coefficients $a_{i}$ and $b_{i}$ are all real numbers. It will be assumed still further that the system is a stable one. That is, if the input signal is removed, the output signal becomes arbitrarily small with increasing time. This restricts the denominator $D(s)$ to being a Hurwitz polynomial (a polynomial whose roots all have negative real parts). Finally, the transfer function of any physically realizable system must become arbitrarily small as the magnitude of the complex frequency $s$ becomes arbitrarily large. This is due to the stray capacities and parameter dissipation that must exist in such a system. Thus, this discussion will be restricted, in addition, to those transfer functions that have more poles than zeros $(m>n)$. This insures that the Fourier transform, relating the transfer function $Z(j \omega)$ to its corresponding unit impulse response $W(t)$, exists. Only those transfer functions which satisfy the aforementioned conditions will be considered in this paper. Therefore, all unit impulse responses will be a finite sum of terms of the form,

$$
A t^{\mu} e^{-\alpha t} \cos (\beta t+\gamma),
$$

where $A, \alpha, \beta$, and $\gamma$ are constants with $\alpha>0$ and $\mu$ is either zero or a positive integer.

In a previous paper [1], certain classes of these transfer functions have been investigated and many restrictions on the transient responses corresponding to such transfer functions were shown to exist. These classes are related to the class of positive real functions for which $m-n$ equals one. However, the sum of all these classes does not include every transfer function of the type considered here. A transfer function that belongs to one of these classes is called a class $k$ function and the integer $k$ identifies the particular class. Throughout this paper, the positive integer $k$ will represent the number of poles in excess of the number of zeros.

The purpose of this paper is to determine other properties of the class $k$ functions and then to extend the restrictions on their corresponding transient responses to the

*Received June 25, 1958. 
transient responses of any transfer function. That is, a means will be developed for determining bounds on the transient responses of any transfer function, whether or not that transfer function is a class $k$ function, by relating it to a class $k$ function.

The class $k$ functions. For this investigation, the form of the definition of class $k$ functions will be altered somewhat from that given in [1]. It should be emphasized, however, that these two definitions are the same in that they define exactly the same classes of functions. The new definition leads to simpler expressions in this paper.

Consider the function $Z_{a}(s)$ which is obtained by successively integrating the transfer function $Z(s)$ according to $(2)$

$$
Z_{a}(s)=\int_{s}^{\infty} d s_{a-1} \int_{s_{q-1}}^{\infty} d s_{a-2} \cdots \int_{s_{1}}^{\infty} Z\left(s_{0}\right) d s_{0} .
$$

The integer $q$ is restricted to being less than or equal to the integer $k-1$. The righthand side of (2) is, in general, a multivalued function and, if all the poles of $Z(s)$ are simple, it may be written as

$$
(-1)^{a} K \sum_{i=1}^{m} k_{i} \frac{\left(s+\rho_{i}\right)^{q-1}}{(q-1) !}\left[\ln \left(s+\rho_{i}\right)-\sum_{p=1}^{q-1} \frac{1}{p}\right]
$$

where the $\rho_{i}$ are the poles of $Z(s)$ and the $k_{i}$ are the residues of these poles. [ $Z_{q}(s)$ will be single-valued when $Z(s)$ has only one pole of multiplicity $m$.] For the purposes of this paper, only one branch of this multivalued function is needed. Therefore, given any $Z(s)$ having at least two more poles than zeros, branch cuts consisting of straight line segments connecting all the poles will be assumed. In addition, these straight line segments will be so chosen that any pair of poles can be connected by a path traced exclusively along these segments. That is, it will be assumed that these branch cuts form a tree with the distinct poles at its vertices. The number of these branch cuts will be one less than the number of distinct poles of $Z(s)$. Finally, it will be assumed throughout this paper that the paths of integration in (2) never cross any of these branch cuts. Under these conditions, (2) defines a single-valued function $Z_{a}(s)$.

Representing the real and imaginary parts of $s_{q}$ and $Z_{q}(j \omega)$ by $s_{q}=\sigma_{q}+j \omega_{q}$ and $Z_{a}(j \omega)=R_{a}(\omega)+j I_{a}(\omega)$, the real part of $Z_{a}(j \omega)$ is given by the following expressions. For $q$ odd,

$$
R_{a}(\omega)=(-1)^{(a+1) / 2} \int_{\omega}^{\infty} d \omega_{a-1} \int_{\omega_{a-1}}^{\infty} d \omega_{a-2} \cdots \int_{\omega_{1}}^{\infty} I\left(\omega_{0}\right) d \omega_{0}
$$

and for $q$ even,

$$
R_{a}(\omega)=(-1)^{a / 2} \int_{\omega}^{\infty} d \omega_{a-1} \int_{\omega_{a-1}}^{\infty} d \omega_{a-2} \cdots \int_{\omega_{1}}^{\infty} R\left(\omega_{0}\right) d \omega_{0},
$$

where $R(\omega)$ and $I(\omega)$ are the real and imaginary parts of $Z(j \omega)$, respectively.

The class $k$ functions may now be defined as follows.

Definition. The transfer function $Z(s)$ will be called a class $k$ function, where $k=m-n$, if $Z_{k-1}(s)$ is a positive real function in the half plane $\sigma \geq 0$.

An immediate consequence of this definition is easily obtained. The necessary and sufficient condition for $Z(s)$ to be a class $k$ function is that $R_{k-1}(\omega) \geq 0$ for all $\omega$. This follows from the definition since $Z_{k-1}(s)$ is an analytic function and, moreover, $Z_{k-1}(\sigma)$ is a real function for $\sigma \geq 0$. 
Furthermore, class $k$ functions may be generated from the class 1 functions by differentiation. More generally, if $Z(s)$ is class $k$, then $(-1)^{h} d^{h} Z / d s^{h}$ is class $(k+h)$. Another elementary property of these functions is that $\alpha Z_{a}(s)+\beta Z_{b}(s)$ is class $k$ if $Z_{a}(s)$ and $Z_{b}(s)$ are each at least class $k$ and $\alpha$ and $\beta$ are positive numbers.

Realizability conditions on the impulse response. It will be assumed throughout this paper that all driving functions of time are applied at $t=0$. The unit impulse response $W(t)$ is, therefore, related to the transfer function $Z(s)$ through the Laplace transform,

$$
Z(s)=\int_{0}^{\infty} W(t) e^{-s t} d t
$$

where $\sigma$ is greater than the real part of any pole of $Z(s)$. Moreover, the function $Z_{a}(s)$ may be obtained by integrating (5) under the integral sign $q$ times. The resulting expression (6) is valid for $q \leq k-1$ [2, Theorem 17, p. 273].

$$
Z_{a}(s)=\int_{0}^{\infty} \frac{W(t)}{t^{a}} e^{-s t} d t
$$

A well known result of circuit theory is that, if $Z(s)$ is a positive real function, then it is expressible in terms of three positive definite quadratic forms which are related to the total stored energy and power dissipation in the network [3, Chap. 4]. There is a somewhat similar result in the theory of Laplace transform which will place restrictions on the unit impulse response corresponding to a positive real $Z(s)[4$, Theorem 6]. More precisely, $Z(s)$ is a class 1 function if and only if $W_{0}(t)$ is a positive definite function where $W_{c}(t)$ is the even function given by

$$
W_{e}(t)= \begin{cases}W(t) & \text { for } t \geq 0 \\ W(-t) & \text { for } \quad t \leq 0 .\end{cases}
$$

By definition, $W_{e}(t)$ is positive definite if the Hermitian form

$$
\sum_{i=1}^{n} \sum_{j=1}^{n} W_{e}\left(t_{i}-t_{j}\right) x_{i} x_{i}
$$

is non-negative for all values of the real numbers $t_{1}, t_{2}, \cdots, t_{n}$ and $x_{1}, x_{2}, \cdots, x_{n}$ and for all $n$.

Applying these known results to expression (6), the following realizability theorem is immediately obtained.

Theorem 1. The necessary and sufficient condition for $Z(s)$ to be a class $k$ function is that $W_{e}(t) /|t|^{k-1}$ be a positive definite function. That is, $Z(s)$ is class $k$ if and only if

$$
\sum_{i=1}^{n} \sum_{i=1}^{n} \frac{W_{e}\left(t_{i}-t_{i}\right)}{\left|t_{i}-t_{i}\right|^{k-1}} x_{i} x_{i} \geq 0
$$

for all values of real numbers $t_{1}, t_{2}, \cdots t_{n}$ and $x_{1}, x_{2}, \cdots, x_{n}$ and for all $n$.

There are several necessary and sufficient conditions for a function to be positive definite [4 through 8] which could similarly be used for realizability conditions. However, all of these are, in general, too complicated to be useful as a practical test.

A condition which is sufficient (though not necessary) and at the same time quite 
simple to apply may be obtained by applying Theorem 5 of [9] to the function $W(t) / t^{k-1}$ for the case where the integer $m$, defined in that theorem, equals one. This yields the following sufficiency test for a class $k$ function.

Theorem 2. If the second derivative of $W(t) / t^{k-1}$ is non-negative for $t>0$, then $Z(s)$ will be a class $k$ function.

A necessary but not sufficient condition on the impulse response, in order that it be the Laplace transform of a class $k$ function was given in [1, Theorem 9]. A new proof, which is considerably simpler than the one given previously, may be constructed by using an operation transform pair of the Laplace transform.

Theorem 3. If $Z(s)$ is class $k$, then, for $t \geq 0$,

$$
|W(t)| \leq \frac{K t^{k-1}}{(k-1) !}
$$

Proof. By the definition of class $k$ functions, the function $Z_{k-1}(s)$ is positive real and the first term of its inverse series expansion is

$$
\frac{K}{(k-1) ! s},
$$

where $k=m-n$. It has been shown that such a positive real function has a unit impulse response which is bounded by the coefficient of the first term in its inverse series expansion [9, Theorem 1]. Moreover, each integration in expression (2) corresponds to the division of $W(t)$ by the time variable $t$. This transformation of the operations of complex integration and division by $t$ is a well-known property of the Laplace transformation [2, Theorem 17, p. 273]. Thus, for $t \geq 0$,

$$
\left|\frac{W(t)}{t^{k-1}}\right| \leq \frac{K}{(k-1) !},
$$

which is the desired conclusion.

By use of the superposition integral, the previous result may be extended to those transfer functions that are products of class $k$ functions. This is applicable, for instance, to a system of cascaded amplifiers each of whose interstage networks is class $k$. It may also be applied to the transfer functions of ladder networks [10].

Theorem 4 . If the functions $A(s), B(s), \cdots, H(s)$ are class $a$, class $b, \cdots$, and class $h$, respectively, and their constant multipliers are $K_{a}, K_{b}, \cdots$, and $K_{h}$, respectively, then the unit impulse response $W(t)$ corresponding to the product of these functions, $A(s) B(s) \cdots$ $H(s)$, is bounded by

$$
|W(t)| \leq \frac{K_{a} K_{b} \cdots K_{h}}{(a+b+\cdots+h-1) !} t^{(a+b+\cdots+h-1)}
$$

for $t \geq 0$.

Proof. Let $W_{a b}(t)$ be the inverse Laplace transform of the product $A(s) B(s)$. By the superposition integral,

$$
\left|W_{a b}(t)\right| \leq \int_{0}^{t}\left|W_{a}(\tau)\right|\left|W_{b}(t-\tau)\right| d \tau .
$$


But, since $A(s)$ is class $a$ and $B(s)$ is class $b$,

$$
\left|W_{a b}(t)\right| \leq \frac{K_{a} K_{b}}{(a-1) !(b-1) !} \int_{0}^{t} \tau^{a-1}(t-\tau)^{b-1} d \tau .
$$

Integrating this expression by parts $b-1$ times,

$$
\left|W_{a b}(t)\right| \leq \frac{K_{a} K_{b}}{(a+b-2) !} \int_{0}^{t} \tau^{a+b-2} d \tau=\frac{K_{a} K_{b}}{(a+b-1) !} t^{(a+b-1)} .
$$

Applying this process repeatedly to the sequence of products $A(s) B(s), A(s) B(s) C(s)$, $\cdots, A(s) B(s) \cdots H(s)$, expression (11) will be obtained.

A relationship between an arbitrary transfer function and the class $k$ functions. One of the objects of this paper is to develop a method of determining bounds on the transient responses of any transfer function satisfying the restrictions enumerated in the introduction. This will be accomplished by relating such a transfer function to a class $k$ function as follows.

Theorem 5. For any given $Z(s)$, a real number $c$ can be found such that $Z(s+c)$ is class $k$. Moreover, if $Z(s+c)$ is class $k$, then $Z(s+d)$ is class $k$ for all d greater than $c$.

Proof. By the definition of class $k$ functions, $Z(s)$ will be class $k$ only if $Z_{k-1}(s)$ is a positive real function. Now consider the loci of $\operatorname{Re}\left[Z_{k-1}(s)\right]=0$. Since $Z_{k-1}(s)$ behaves as $1 / s$ in the neighborhood of $s=\infty$, only one such locus exists in a sufficiently small neighborhood of $s=\infty$. Moreover, this locus is asymptotic to a line parallel to the imaginary axis. Thus, for $c$ sufficiently large, the line $s=c+j \omega$, which is parallel to the imaginary axis, will not intersect any locus of $\operatorname{Re}\left[Z_{k-1}(s)\right]=0$. Furthermore, $\operatorname{Re}[1 / s] \geq 0$ for $\sigma \geq 0$. Therefore, $\operatorname{Re}\left[Z_{k-1}(s)\right]>0$ for $\sigma \geq c$.

The second statement of this theorem follows from the fact that a positive real function of a positive real function is positive real.

Theorem 6. If the real number $c$ is such that $Z(s+c)$ is class $k$, then $c$ cannot be less than $\left(a_{n-1}-b_{m-1}\right) / k$, where $a_{n-1}$ and $b_{m-1}$ are the coefficients indicated in (1). Moreover. $c$ cannot be less than the real parts of all the poles of $Z(s)$.

Proof. Integrating the inverse series expansion of $Z(s)$ according to (2), a series expansion for $Z_{k-1}(s)$ may be found

$$
Z_{k-1}(s)=\frac{K}{k !}\left(\frac{k}{s}+\frac{a_{n-1}-b_{m-1}}{s^{2}}+\cdots\right) .
$$

It can be seen from the first two terms that a locus of $\operatorname{Re}\left[Z_{k-1}(s)\right]=0$ is asymptotic to the line,

$$
s=\frac{a_{n-1}-b_{m-1}}{k}+j \omega,
$$

for $\omega$ sufficiently large. Moreover, such loci are continuous curves which pass through the singularities of $Z_{k-1}(s)$. Since all the singularities of a positive real function are excluded from the right half $s$ plane, the constant $c$ cannot be less than $\left(a_{n-1}-b_{m-1}\right) / k$ if the line $s=c+j \omega$ is not to intersect a locus of $\operatorname{Re}\left[Z_{k-1}(s)\right]=0$ and if $Z_{k-1}(s+c)$ is to be analytic in the right half $s$ plane. Moreover, the singularities of $Z_{k-1}(s)$ occur at the same points as the poles of $Z(s)$ so that $c$ cannot be less than the real part of any such pole. 
One physical interpretation of the addition of a positive constant $c$ to the complex frequency variable $s$ is that dissipation is added uniformly to all the reactive elements of any network which realizes $Z(s)$. In particular, every inductance $L_{i}$ has a resistance $R_{i}=L_{i} c$ inserted in series with it and every condenser $C_{i}$ has a conductance $G_{i}=C_{i}$ $c$ connected in parallel with it [3, pp. 706-708].

A class $k$ function $Z(s+c)$ can be obtained from a given $Z(s)$ in the following way. Consider the polynomial in the numerator of $Z(s)$

$$
N(s)=s^{n}+a_{n-1} s^{n-1}+\cdots+a_{1} s+a_{0} .
$$

The polynomial $N(s+c)$ may be determined by setting $\mu$ equal to $s+c$ and expanding $N(\mu)$ into a Taylor series around the point $\mu=c$

$$
\left.\left.N(s+c)=s^{n}+\frac{s^{n-1}}{(n-1) !} \cdot \frac{d^{n-1} N(s)}{d s^{n-1}}\right]_{s-c}+\cdots+s \frac{d N(s)}{d s}\right]_{s=c}+N(c) .
$$

A simple method exists for obtaining the coefficients of $N(s+c)$ from the coefficients of $N(s)$ without having to perform the differentiations indicated in (13) [11, pp. 52-54]. This procedure is especially useful when a numerical value for $c$ is used. Divide $N(s)$ by $s-c$ until a constant remainder $r_{0}$ is obtained.

$$
\frac{N(s)}{s-c}=Q_{n-1}(s)+\frac{r_{0}}{s-c} .
$$

The value of $N(c)$ is $r_{0}$. Dividing the quotient $Q_{n-1}(s)$ in (14) by $s-c$ until another constant remainder $r_{1}$ is obtained, the value of the first derivative of $N(s)$ at $s=c$ will be found.

$$
\begin{gathered}
\frac{Q_{n-1}(s)}{s-c}=Q_{n-2}(s)+\frac{r_{1}}{s-c}, \\
\left.\frac{d N(s)}{d s}\right]_{s=c}=r_{1} .
\end{gathered}
$$

Repeating this process, the value of the $i$ th derivative of $N(s)$ at $s=c$ divided by factorial $i$ is found to be equal to the $i$ th constant remainder $r_{i}$

$$
\left.\frac{1}{i !} \cdot \frac{d^{i} N(s)}{d s^{i}}\right]_{s=c}=r_{i} .
$$

The verification of these relations may be found in [11, Chap. 3].

Denoting the remainder, obtained from the denominator $D(s)$ through the procedure indicated above, by $r_{i}^{\prime}$, the expression for $Z(s+c)$ may be written as

$$
Z(s+c)=K \frac{s^{n}+r_{n-1} s^{n-1}+\cdots+r_{1} s+r_{0}}{s^{m}+r_{m-1}^{\prime} s^{m-1}+\cdots+r_{1}^{\prime} s+r_{0}^{\prime}} .
$$

Therefore, for any given $Z(s)$, a $c$ may be determined which produces a class $k$ function $Z(s+c)$ by applying the tests for a class $k$ function [1, Theorems 4 and 6 ] to expression (15).

If $Z(s)$ has only one finite zero, an application of [1, Theorem 5] yields values for $c$ which insure that $Z(s+c)$ is class $k$. In this case, $Z(s+c)$ has the form

$$
Z(s+c)=K \frac{s+c+a_{0}}{s^{m}+r_{m-1}^{\prime} s^{m-1}+\cdots+r_{1}^{\prime} s+r_{0}^{\prime}}=K \frac{s+c+a_{0}}{D(s+c)},
$$


where $r_{m-1}^{\prime}=m c+b_{m-1}, r_{1}^{\prime}=d D(s) / d s$ at $s=c$, and $r_{0}^{\prime}=D(c)$. For $m$ even, $Z(s+c)$ will be class $m-1$ for all values of $c$ greater than or equal to $\left(a_{0}-b_{m-1}\right) /(m-1)$. For $m$ odd, this theorem places two restrictions on $c$; it must be greater than or equal to $\left(a_{0}-b_{m-1}\right) /(m-1)$ and greater than $\left(r_{0}^{\prime} / r_{1}^{\prime}-a_{0}\right)$.

A simple graphical method for determining this constant $c$ to insure a class $k$ function $Z(s+c)$ is based on the fact that, if the phase angle of $Z(j \omega)$ equals an odd multiple of $\pi / 2$ exactly $k-1$ times for $k$ odd or if this angle equals a multiple of $\pi$ or zero exactly $k-1$ times for $k$ even and if $d \varphi / d \omega<0$ at $\omega=0$, then $Z(s)$ is class $k$ [1, p. 282]. The procedure is quite simple if $N(s)$ and $D(s)$ are factored

$$
Z(s)=K \frac{\left(s-\mu_{1}\right)\left(s-\mu_{2}\right) \cdots\left(s-\mu_{n}\right)}{\left(s-\rho_{1}\right)\left(s-\rho_{2}\right) \cdots\left(s-\rho_{m}\right)} .
$$

Following the convention that the phase angle of any factor in (17) remains within the interval $(3 \pi / 2,-\pi / 2)$, the phase angle of $\left(s-\mu_{i}\right)$ will be denoted by $\psi_{i}$ and the phase angle of $\left(s-\rho_{i}\right)$ will be denoted by $\theta_{i}$. The phase angle $\varphi$ for $Z(s+c)$ is then given by

$$
\varphi=\sum_{i=1}^{n} \psi_{i}-\sum_{i=1}^{m} \theta_{i}
$$

Now the phase angle of the factor $\left(s+c-\mu_{i}\right)$ is the angle of the vector from the point $\mu_{i}$ to the point $s+c$. Consequently, the variations of $\varphi$ along a series of lines parallel to the imaginary axis in the $s$ plane may be obtained graphically by applying (18). For some sufficiently large value of $c$, the aforementioned sufficiency criterion on the values of $\varphi$ for $s=j \omega$ will be fulfilled and, for this value of $c, Z(s+c)$ will be class $k$.

Bounds on the impulse response of any transfer function. Now that any transfer function $Z(s)$ may be related to a class $k$ function $Z(s+c)$ by an appropriate choice of $c$, the restrictions on the impulse responses of class $k$ functions may be extended to the impulse response of $Z(s)$.

Theorem 7. The unit impulse response $W(t)$ corresponding to any transfer function $Z(s)$ is bounded by

$$
|W(t)| \leq \frac{K e^{c t} t^{k-1}}{(k-1) !}
$$

where the constant $c$ is such that $Z(s+c)$ is a class $k$ function.

Proof. Consider the inverse Laplace transformation between $Z(s)$ and $W(t)$ where the path of integration is along the line $s=c+j \omega$

$$
W(t)=\frac{1}{2 \pi j} \int_{c-i \infty}^{c+i \infty} Z(s) e^{s t} d s
$$

Making the change of variable $s=v+c,(20)$ becomes

$$
W(t)=e^{c t} W_{c}(t),
$$

where

$$
W_{c}(t)=\frac{1}{2 \pi j} \int_{-j \infty}^{+j \infty} Z(v+c) e^{v t} d v
$$


Since $Z(v+c)$ is a class $k$ function of $v$, Theorem 3 may be invoked to complete the proof. It should be noted that $c$ need not be a positive number if $Z(s)$ is class $k$.

An application of $[1$, Theorem 10$]$ to the quantity $W(t) / e^{c t}$ yields still another restriction on the unit impulse response of any transfer function.

Theorem 8. Let $\left|W(t) e^{-c t}\right|$ be less than or equal to the positive constant $M$ for $t \geq \tau$. For any transfer function $Z(s)$, let $c$ be such that the imaginary part of $Z_{k-2}(c+j \omega)$ is non-positive for $\omega \geq 0$. Then, for $0 \leq y<1$,

$$
|W(y \tau)| \leq e^{c y \tau}\left[\frac{K(y \tau)^{k-2} \sin \pi y}{\pi(k-1) !}+\frac{2 M y^{k} \sin \pi y}{\pi} \sum_{p=1}^{\infty} \frac{1}{p^{k-1}\left(p^{2}-y^{2}\right)}\right] .
$$

Appendix. As an illustration, the bounds on the unit impulse response of the transfer function,

$$
Z(s)=\frac{s+\mu}{(s+\rho)^{3}},
$$

will be obtained. The only restriction imposed upon the real numbers $\mu$ and $\rho$ is that $\rho$ is positive. The unit impulse response corresponding to $Z(s)$ is

$$
W(t)=e^{-\rho t}\left[t+\frac{\mu-\rho}{2} t^{2}\right] .
$$

Now, the lowest real number $c$ which makes $Z(s+c)$ a class 2 function will be found. According to the definition, $Z(s+c)$ will be class 2 when $Z_{1}(s+c)$ is positive real. But,

$$
Z_{1}(s)=\int_{0}^{\infty} \frac{s_{0}+\mu}{\left(s_{0}+\rho\right)^{3}} d s_{0}=\frac{s+\frac{\mu+\rho}{2}}{(s+\rho)^{2}} .
$$

Calculating the real part of $Z_{1}(s+c)$, it can be seen that this real part is non-negative only when $c$ satisfies the following inequalities

$$
\begin{gathered}
c \geq-\frac{\mu+\rho}{2} \text { for } \mu \leq \rho, \\
c \geq \frac{\mu-3 \rho}{2} \text { for } \mu \geq \rho .
\end{gathered}
$$

Thus, by Theorem 7

$$
|W(t)| \leq t \exp \left(-\frac{\mu+\rho}{2} t\right) \text { for } \mu \leq \rho
$$

and

$$
|W(t)| \leq t \exp \left(\frac{\mu-3 \rho}{2} t\right) \text { for } \mu \geq \rho .
$$

By comparison with expression (23), it is easy to show that these inequalities are indeed true. 


\section{REFERENCES}

1. A. H. Zemanian, On transfer functions and transients, Quart. Appl. Math. 16, 273-294 (1958)

2. M. F. Gardner and J. L. Barnes, Transients in linear systems, vol. 1; John Wiley and Sons, New York, 1942

3. D. F. Tuttle, Jr., Network synthesis, vol. 1, John Wiley and Sons, New York, 1958

4. M. Mathias, Uber positive Fourier-Integrale, Math. Z. 16, 103-125 (1923)

5. M. Ky Fan, Les fonctions definies-positives et les fonctions completement monotones, Mem. Sci. Math., Acad. Sci. Paris, Fascicule 64 (1950)

6. H. Cramer, On the representation of a function by certain Fourier integrals, Trans. Am. Math. Soc. 46, 191-201 (1939)

7. S. Bochner, Monotone Funktionen, Stieltjessche Integrale und harmonische Analyse, Math. Ann. 108, 378-410 (1933)

8. K. Yosida, On the representation of functions by Fourier integrals, Proc. Imperial Acad., Tokyo, 20, 655-660 (1944)

9. A. H. Zemanian, Bounds existing on the time and frequency responses of various types of networks, Proc. IRE 42, 835-839 (1954)

10. A. H. Zemanian and P. E. Fleischer, On the transient responses of ladder networks, Trans. IRE, PGCT CT-5, 197-201 (1958)

11. L. Weisner, Introduction to the theory of equations, The Macmillan Co., New York, 1938 